\title{
GLYCEROL DERIVATIVES AS A MODERN PLASTICIZERS FOR STARCH FILMS
}

\author{
Zuzanna Żołek-Tryznowska, Łukasz Cichy \\ Warsaw University of Technology, Faculty of Production Engineering, \\ Mechanics and Printing Institute, Warsaw, Poland
}

\begin{abstract}
Due ecological reasons there is a considerable interest in biodegradable films made from renewable and natural polymers, such as starch. Starch films are not meant to totally replace conventional packaging polymer films, but they might be a competition in several application, where traditional packaging cannot function. Moreover, starch as a raw material exhibit many benefits, such as relatively low price, abundant, biodegradable, and edible. Films prepared from starches are isotropic, odourless, tasteless, colourless, non-toxic and biodegradable. Finally, they are nutritious and safe, so they are used in the marketing of food. Unfortunately, the starch films present poor tensile properties and sensitivity to moisture content.

In order to modify starch films properties the addition of various plasticizers is used. Commonly as a plasticizer water and glycerine is used. In this work, we use glycerol derivatives as a modern plasticizers for starch films obtained from potato starch by the tape casting technique, which allows to obtained films with constant thickness and $20 \mathrm{~cm}$ length. The influence of the ratio of various plasticizers: glycerol, pentaerythiolethoxylate, glycerol ethoxylate and Poligliceryn-3 on the mechanical properties and surface free energy was investigated. The plasticizers selected by us are characterized by a large number of functional hydroxyl groups. The starch films with the mixture of plasticisers reveal better usable properties and higher mechanical properties than with only one plasticizer. However, glycerol derivatives they cannot be used without the addition of glycerol. Furthermore, surface free energy was determinate by OwensWendt and van Oss-Chaudhury-Good method. The values of SFE are in the range $50-60 \mathrm{~mJ} / \mathrm{m}^{2}$ and higher than values of typical plastic films, which are used in packaging industry. Obtained starch films are characterized by quite high polar component of SFE, with may be related with the influence of hydroxyl groups.

Our results support the use of glycerol derivatives as modern plasticizers for biodegradable films and may open up new possibilities for applications of these compounds.
\end{abstract}

Key words: biodegradable films, starch, surface free energy, glycerol

\section{INTRODUCTION}

Plastic are commonly used in various industries, however one of the main recipient of them is packaging industry. In Poland the usage of plastics as a flexible films is estimated at around $13 \mathrm{~kg}$ per person per year. Furthermore, in the packaging industry only six plastics (HDPE, LDPE, PVC, PP, PS and PET) are used. Due their great packaging properties, such as good mechanical properties, gas and water barrier, plastic films have found application as excellent materials for packaging of food products. Plastic films are used in the packaging industry usually as single-use packaging, which means that after consuming, they must be recycled.

The main disadvantage of conventional plastic materials is the method of recycling. Utilization of plastic films is a problem due their non-degradability in natural environment, hence the European Union introduce the regulation of single-use plastics. In order to decrease the usage of conventional plastics, bio-based and biodegradable plastics are introduced. Some of the environmentally friendly polymers are already industrially produced (PLA, PHA, PCL, PEA), however the researchers search for new, cheap, with good properties plastics based on renewable and natural raw materials, for example polysaccharides (Lawton, 1996). Furthermore, the price of biodegradable PLA is still higher than conventional nondegradable PP or PET.

Starch is one of the most abundant naturally occurring substances (Pareta et al, 2006). Furthermore, it exists in various forms depending on the origin of the raw material (Sartori et al, 2016). Depending on origin of starch, give rise to a variety of films. The films based on maize (Pareta et al, 2006), banana (Sartori et al, 2016), cassava (Bourtoom et al, 2008, Souza et al, 2013), potato starch (Talja et al, 2007), have been already presented in the literature. The most widely used technique for the laboratorial production of starch-based films is casting method (Vicentini et al, 2002; Mali et al, 2005a; Müller et al, 2008). The suspension of starch in water is poured on Petri dish, while the thickness of the resulting films from the mass of suspension 
poured on the plate. The film is drying at room temperature or in ovens with forced air. This method exhibit some disadvantages: (i) uncontrolled thickness of the starch film, (ii) long time of drying (24 h or more), (iii) limited and small size of obtained starch film, which enables to scale up the production to industrial scale. Addition of plasticizers to the starch films affects the properties such as water sorption, mechanical properties and glass transition temperature. Generally, as a plasticizers glycerine is used. However, also some of polyols, (sorbitol, xylitol) may be used as plasticizers (Talja et al., 2007). Furthermore, water sorbed in film acts also as plasticizer.

The purpose of this work was to found novel application of glycerol derivatives, as plasticizers for starch films. In this work we use potato starch, because is the most common and cheapest starch. As a plasticizers various glycerol derivatives (pentaerythiolethoxylate, glycerol ethoxylate and Poligliceryn-3) were used. Additionally, we have improved well-known casting method, which allows to control the thickness of obtained films. The influence of the ratio of various plasticizers on surface free energy was investigated. Surface free energy was calculated using two various methods: Owens-Wendt and van Ossy-Chaudhury-Good.

\section{METHODS}

The properties of glycerol derivatives are shown in Table 1. Table 2 shows the formulation of solution used for forming the starch films.

Table 1: Properties of glycerine and its derivatives

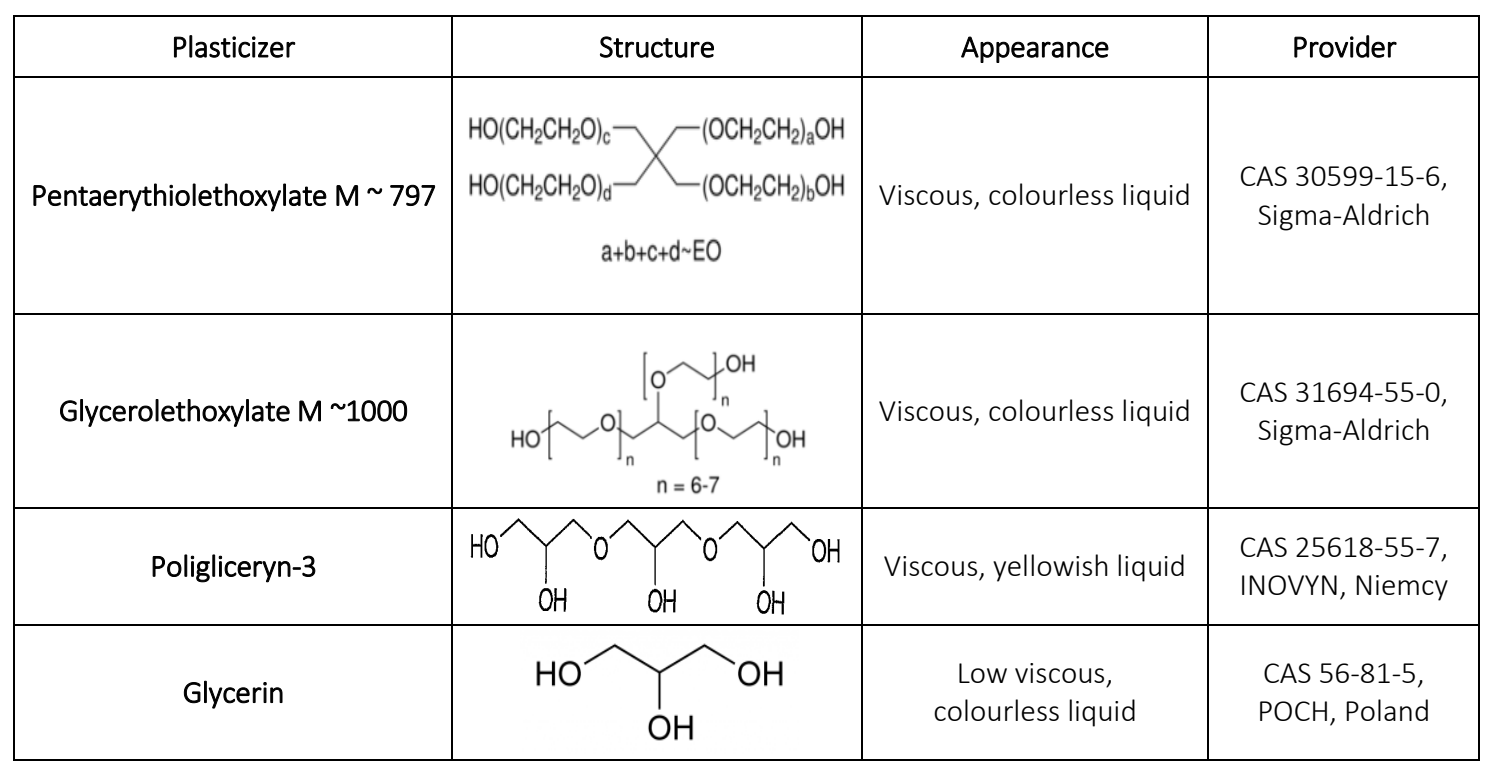

Amount of glycerol or glycerol derivative was added into a solution of $4 \mathrm{~g}$ of starch in $100 \mathrm{~g}$ of water. Next, the film forming suspension was heated with continuous mixing over $95^{\circ} \mathrm{C}$ and kept for 5 min before letting it cool down to $50^{\circ} \mathrm{C}$ to obtain the film forming solution. Film forming solution was casted on a Tefloncoated plate placed on a K Paint applicator equipped in a spreader with gap $3 \mathrm{~mm}$. The films were dried in a room temperature and humidity $30 \% \mathrm{RH}$.

Table 2: Formulation of investigated starch films

\begin{tabular}{|c|c|c|c|c|c|}
\hline Sample & Starch [g] & Glycerol [g] & Poliglycerol-3 [g] & Pentaerythiolethoxylate [g] & Glycerolethoxylate [g] \\
\hline 1 & 4 & 1.6 & 0.8 & - & - \\
\hline 2 & 4 & 2.8 & 0.4 & - & - \\
\hline 3 & 4 & 1.6 & - & 0.8 & - \\
\hline 4 & 4 & 1.6 & - & - & 0.8 \\
\hline 5 & 4 & 2.8 & - & - & 0.4 \\
\hline 6 & 4 & 2.8 & - & 0.4 & - \\
\hline
\end{tabular}


Contact angle of the obtained films was measured using a DSA 30 E drop shape analysis system (Krüss, Germany). In order to measure contact angle, smooth and horizontal sessile drops of the liquids were deposited on a solid surface using needles of $0.5 \mathrm{~mm}$ diameter. The contact angle was measured on static drops. The drop shape analysis was done $15 \mathrm{~s}$ after the drop deposition with Tangent method 2. Environmental conditions were stable with temperature $23 \pm 0.5^{\circ} \mathrm{C}$. The reported contact angle values are the mean of 6 probes on two films. The SFE was calculated using Owens-Wendt and van-OssyGood method.

Testing of mechanical properties of obtained starch films was performed using wick BT1-FB010TN.D30 tensometer (Zwick-Roell, Germany). Samples for the tests were prepared conforming to ISO 527-1. The width of the strip was $10 \mathrm{~mm}$ and the length was $100 \mathrm{~mm}$ for all tested films, and initial distance of the clamps was set to $50 \mathrm{~mm}$. The speed of stretching was $100 \mathrm{~mm} \mathrm{~min}^{-1}$. Tensile strength and elongation properties were determined from the stressestrain curves. For each film, at least 3 samples from two various films were tested and the mean values were taken as the final result.

\section{RESULTS}

We have prepared about 23 various starch films based on differ properties of plasticizers. However, the quality of some of them did not allowed for surface free energy and mechanical properties measurements. The properties of obtained films are presented in Table 3.

Table 3: Organoleptic properties of formed starch films

\begin{tabular}{|c|c|c|c|c|}
\hline Sample & Colour & Smell & Flexibility & Extensibility \\
\hline $\mathbf{1}$ & Transparent & Odourless & Flexible & Slightly stretchable \\
\hline $\mathbf{2}$ & Transparent & Odourless & Very flexible & Very stretchable \\
\hline $\mathbf{3}$ & Transparent & Odourless & Slightly flexible & Non stretchable \\
\hline $\mathbf{4}$ & Transparent & Odourless & Slightly flexible & Non stretchable \\
\hline $\mathbf{5}$ & Transparent & Odourless & Flexible & Stretchable \\
\hline $\mathbf{6}$ & Transparent & Odourless & Flexible & Stretchable \\
\hline
\end{tabular}

The values of contact angle and surface free energy are listed in Table 4. The values of surface free energy (SFE) calculated by Owens-Wendt method and van Ossy-Good-Chaudhury method are listed in Table 5 and Table 6, respectively.

Table 4: Contact angles and SFE calculated using Owens-Wendt method of obtained films

\begin{tabular}{|c|c|c|c|}
\hline Sample & Water $\left[^{\circ}\right]$ & Diiodomethane $\left[^{\circ}\right]$ & Formamide $\left[^{\circ}\right]$ \\
\hline 1 & 46.50 & 32.26 & 32.88 \\
\hline 2 & 35.10 & 34.54 & 27.10 \\
\hline 3 & 45.37 & 20.43 & 36.67 \\
\hline 4 & 15.85 & 22.64 & 26.95 \\
\hline 5 & 29.32 & 32.83 & 37.96 \\
\hline 6 & 24.94 & 32.10 & 31.66 \\
\hline
\end{tabular}

Table 5: Values of SFE together with polar and dispersive components calculated using Owens-Wendt method

\begin{tabular}{|c|c|c|c|}
\hline Sample & Dispersive component $\left[\mathrm{mJ} \bullet \mathrm{m}^{-2}\right]$ & Polar component $\left[\mathrm{mJ} \bullet \mathrm{m}^{-2}\right]$ & SFE $\left[\mathrm{mJ} \cdot \mathrm{m}^{-\mathbf{2}}\right]$ \\
\hline 1 & 43.3 & 18.5 & 61.8 \\
\hline 2 & 42.2 & 25.2 & 67.4 \\
\hline 3 & 47.7 & 17.3 & 65.0 \\
\hline 4 & 47.0 & 30.5 & 77.4 \\
\hline 5 & 43.0 & 27.6 & 70.6 \\
\hline 6 & 43.3 & 29.3 & 72.7 \\
\hline
\end{tabular}


Table 6: Values of SFE together with its acid-base components and Lifshitz-Van der Waals calculated using van OssyGood-Chaudhury method

\begin{tabular}{|c|c|c|c|c|c|}
\hline Sample & $\gamma^{\mu \mathrm{w}}\left[\mathrm{mJ} \cdot \mathrm{m}^{-2}\right]$ & $V^{+}\left[\mathrm{mJ} \bullet \mathrm{m}^{-2}\right]$ & $V\left[\mathrm{~mJ} \bullet \mathrm{m}^{-2}\right]$ & $\nu^{\mathrm{AB}}\left[\mathrm{mJ} \bullet \mathrm{m}^{-2}\right]$ & SFE $\left[\mathrm{mJ} \bullet \mathrm{m}^{-2}\right]$ \\
\hline 1 & 43.3 & 0.4 & 29.6 & 7.0 & 50.3 \\
\hline 2 & 42.2 & 0.5 & 40.5 & 9.3 & 51.6 \\
\hline 3 & 47.7 & 0.0 & 34.1 & 0.6 & 48.3 \\
\hline 4 & 47.0 & 0.0 & 60.1 & 0.9 & 47.8 \\
\hline 5 & 43.0 & 0.0 & 56.2 & 1.0 & 44.0 \\
\hline 6 & 43.3 & 0.0 & 55.4 & 3.2 & 46.5 \\
\hline
\end{tabular}

The mechanical properties of obtained films are listed in Table 7.

Table 7: Tensile strength and ultimate elongation of the investigated films

\begin{tabular}{|c|c|c|}
\hline Sample number & Tensile strength [MPa] & Ultimate elongation [\%] \\
\hline $\mathbf{2}$ & 2.06 & 89 \\
\hline $\mathbf{5}$ & 1.76 & 25 \\
\hline $\mathbf{6}$ & 3.92 & 80 \\
\hline
\end{tabular}

\section{DISCUSSION}

We have received about 23 various starch films based on various content of plasticizers. However, some of obtained starch films exhibited fragility and non-flexibility. This films where so fragile that where broken in hands and it was unable to measure contact angle or tensile properties. Only six films where suitable for measurements of contact angle or tensile properties.

The measurements of static contact angles (CA) represent useful information regarding interactions between the coating and the liquid. In general, lower values of CA of water testify to greater wettability of surface and greater hydrophilicity. However, the values of CA of water provide rather greater affinity to water of obtained films.

To the best our knowledge, for the first time surface free energy of starch films were investigated. In this work, in order to calculate SFE two various methods were used: Owens-Wendt (OW) and Van Oss-GoodChaudhury (vOCG). The surface free energy of coatings were evaluated by Owens-Wendt equation:

$\left(1+\cos \theta_{i}\right) \gamma_{l i}=2\left(\sqrt{\gamma_{l i}^{d} \gamma_{s}^{d}}+\sqrt{\gamma_{l i}^{p} \gamma_{s}^{p}}\right)$

where $\vartheta$ is the contact angle (CA) of testing liquid (deg), $\gamma_{l i}$ is the surface free energy (SFE) of the testing liquid, $\gamma_{l i}^{d}, \gamma_{l i}^{p}$ is the dispersion and polar component of tested liquid and finally $\gamma_{s}^{d}, \gamma_{s}^{p}$ are SFE of coating. The acid base theory by van Oss, Good and Chaudhury was used for determination of dispersive, i.e. nonpolar Lifsitz-Van der Waals $\gamma^{\mathrm{LW}}$, acid $\gamma^{+}$, and base $\gamma^{-}$components of the PHU surface energy expressed by terms as acid component $\gamma^{+}$(acceptor effect) and $\gamma^{+}$basic component (donor effect) (Good et al, 1991):

$\left(1+\cos \theta_{i}\right) \gamma_{l i}=2\left(\sqrt{\gamma_{i}^{L W} \gamma_{s}^{L W}}+\sqrt{\gamma_{i}^{L W} \gamma_{s}^{L W}}+\sqrt{\gamma_{i}^{L W} \gamma_{s}^{L W}}\right)$

where $i$ refers to testing liquid and $s$ refers to solid material. The values of SFE calculated with OwensWendt method and van Ossy-Good-Chaudhury method are listed in table 4 and 5, respectively. The values of total SFE calculated by both models, OW and VOCG, differ insignificantly. However, the values of SFE reveals that starch films may be printed without activation process. The highest difference can be observed for sample 3 and is equal nearly $30 \mathrm{~mJ} \cdot \mathrm{m}^{-2}$. The values of Lifshitz-Van der Waals (non-polar) component are much higher than the values of acidic and basic components of SFE. Further, the values of basic component are much higher than values of acidic components. This is related with the presence of electron pairs of oxygen in ether or hydroxyl groups which are effective Lewis base sites. However, compering the acidic 
component, it can be seen that sample 1 and 2 exhibited the highest "acidic" surface character, which may be related with addition of Polyglycerin-3.

Summarizing, all investigated starch films exhibited various values of $\gamma^{\mathrm{AB}}$ and $\gamma^{\mathrm{LW}}$ due various amount of plasticizers and free end-groups (hydroxyl) in the starch and plasticizers, despite their structure is quite similar. The tensile properties of obtained starch films are listed in Table 7. Obtained starch films, in comparison to conventional plastic films, reveal poor tensile properties. However, film with addition of glycerine and Poligliceryn-3 exhibited highest tensile strength.

\section{CONCLUSIONS}

In this work we have demonstrate novel application of glycerol derivatives. The most effective plasticizer for starch films is glycerine and water, however the addition of other glycerine derivatives may improve selected properties of starch films. Unfortunately, the use of other plasticizers or mixture of plasticizers with glycerine requires more research and work. Films that consist more than one plasticizer obtained the best results of surface free energy. The values of surface free energy are higher than the values of surface free energy of conventional plastic films used in packaging. The measurements of SFE shows, that starch films do not requires activation process prior printing. Furthermore, starch films may replace conventional plastics in single-use products, which production may be limited by the regulation of the European Union.

\section{ACKNOWLEDGMENTS}

Funding for this research was provided by the Faculty of Production Engineering, Warsaw University of Technology.

\section{REFERENCES}

[1] Bourtoom, B., Chinnan, M.S.: "Preparation and properties of rice starchechitosan blend biodegradable film" LWT - Food Science and Technology 41 (9), 1633-1641, 2008.

[2] Good R.J., Chaudhury, M.K., van Oss, C.J.: "Theory of Adhesive Forces Across Interfaces" in " Fundamentals of Adhesion" (Springer-Verlag, Boston, USA, 1991) pages 153-172.

[3] Lawton., J.W.: "Effect of starch type on the properties of starch containing films" Carbohydrate Polymers 29 (3), 203-208, 1996.

[4] Mali,. S., Grossmann, M., Garcia, M., Martino, M., Zaritzky, N.: "Mechanical and thermal properties of yam starch films" Food Hydrocolloids 19 (1), 157-164, 2005.

[5] Müller, C., Yamashita. F., Laurindo. J.: "Evaluation of the effects of glycerol and sorbitol concentration and water activity on the water barrier properties of cassava starch films through a solubility approach" Carbohydrate Polymers 72 (1), 82-87, 2008.

[6] Pareta, R., Edirisinghe, M.J.: "A novel method for the preparation of starch films and coatings" Carbohydrate Polymers 63 (3), 425-431, 2006.

[7] Pareta, R., Edirisinghe, M.J.: "A novel method for the preparation of starch films and coatings" Carbohydrate Polymers 63 (3), 425-431, 2006.

[8] Sartori, T., Menegalli, F.C.: "Development and characterization of unripe banana starch films incorporated with solid lipid microparticles containing ascorbic acid" Food Hydrocolloids 55, 210-219, 2016.

[9] Souza, Ac., Goto, G.E.O., Mainardi, J.A., Coelho, A.C.V., Tadini, C.C.: "Cassava starch composite films incorporated with cinnamon essential oil: Antimicrobial activity, microstructure, mechanical and barrier properties" LWT - Food Science and Technology 54 (2), 346-352, 2013.

[10] Talja, R.A., Helén H., Roos. Y.H., Jouppila, K.: "Effect of various polyols and polyol contents on physical and mechanical properties of potato starch-based films" Carbohydrate Polymers 67 (3), 288-295, 2007.

[11] Vicentini, N., Sobral, P., Cereda, M.: "The influence of the thickness on the functional properties of cassava starch edible films" in Plant Biopolymer Science: Food and Non-Food Applications (RSC: Cambridge, UK, 2002) pages 291-300. 


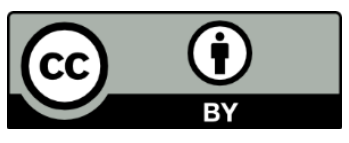

(C) 2018 Authors. Published by the University of Novi Sad, Faculty of Technical Sciences, Department of Graphic Engineering and Design. This article is an open access article distributed under the terms and conditions of the Creative Commons Attribution license 3.0 Serbia

(http://creativecommons.org/licenses/by/3.0/rs/). 